\title{
Short-term exercise training increases plasma levels of klotho and total antioxidant capacity in male Wistar
}

\section{rats}

\author{
Maral Ramez ${ }^{1}$, Farinaz Nasirinezhad ${ }^{2}$, Hamid Rajabi ${ }^{*}$, Nahid Aboutaleb ${ }^{2}$, Nasim Naderi ${ }^{3}$ \\ 'Department of Exercise Physiology, Faculty of Physical Education and Sports Sciences, Kharazmi University, Tehran, Iran \\ ${ }^{2}$ Physiology Research Center and Physiology Department, Faculty of Medicine, Iran University of Medical Sciences, Tehran, Iran \\ ${ }^{3}$ Rajaie Cardiovascular Medical and Research Center, Iran University of Medical Sciences, Tehran, Iran
}

*Corresponding Author: Hamid Rajabi, Faculty of Physical Education and Sports Sciences, Kharazmi University, Tehran, Iran, Email: hrajabi@hotmail.com, Tel: 021-22269549

\begin{abstract}
Background and aims: Soluble or circulating form of Klotho (i.e., anti-aging and longevity protein) has biological effects on various body cells and tissues. Considering the role of exercise training on protective proteins, the present study aimed to investigate and compare the effects of short-term high-intensity interval training (HIIT) and moderate-intensity continuous training (MICT) on plasma levels of Klotho, total antioxidant capacity (TAC), and malondialdehyde (MDA).

Methods: In this study, 24 male Wistar rats with a weight range of 250-300 g and 8-10 weeks old were randomly divided into control, HIIT, and MICT groups. Training included five consecutive days on the treadmill. HIIT including $6 \times 2$ minutes high intermittence with $85 \%-90 \%$ $\mathrm{Vo}_{2}$ max and $5 \times 2$ minutes slow intermittence (active recovery) with $55 \%-60 \% \mathrm{Vo}_{2}$ max. MICT program was performed at $70 \% \mathrm{Vo}_{2}$ max that total distance running was the same for the HIIT group. Then, the plasma levels of Klotho, TAC, and MDA were measured by ELISA assay. Finally, one-way ANOVA and post-hoc Tukey tests were used for statistical analysis.

Results: Based on the results, a significant increase was observed in plasma levels of Klotho and TAC in both HIIT and MICT groups compared to the control group $(P<0.001)$ while these changes were significantly more dominant in the HIIT group compared to MICT group $(P=0.024)$. However, the results demonstrated no significant difference between the groups regarding MDA $(P=0.109)$. Contrarily, the finding revealed a significant positive correlation between Klotho and TAC $(r=0.79,0.83,0.79$ in control, HIIT, and MICT groups, respectively, $P<0.05)$, whereas a significant negative association between Klotho with MDA within all the groups $(r=-0.78,-0.86$, and $-0.81)$.

Conclusion: In general, even short-term aerobic exercise training, especially HIIT increases circulating Klotho and TAC, therefore, this can confirm the positive effects of the training-induced exercise.

Keywords: Exercise training; Klotho; Total antioxidant capacity; High-intensity interval training; Moderate-intensity continuous training
\end{abstract}

Received: 11 March 2018, Accepted: 16 September 2018, ePublished: 28 October 2019

\section{Introduction}

Klotho is an anti-aging and lifespan protein which is mainly expressed in the kidney and brain although lower expression of Klotho is observed in other tissues such as the pituitary gland, parathyroid, skeletal muscle, bladder, ovary, testis, pancreas, thyroid gland, aorta, and blood vessels (1-3). This protein exists in the membrane and soluble forms (2-4). In addition, the circulating soluble form of Klotho is a product of alternative mRNA splicing (i.e., a secreted form of Klotho, $70 \mathrm{kDa}$ isoform). Further, such a soluble form is produced by the proteolytic cleavage related to the extracellular domain of the membrane form $(130 \mathrm{kDa})$ and released into the blood, urine, and cerebrospinal fluid, having biological effects on various body cells and tissues $(3,4)$. In fact, the soluble form of Klotho acts as a hormone and regulates the functions of cells and tissues which express no Klotho, so that the systemic effects of the Klotho are mainly due to the circulating form of this protein $(4,5)$.

Furthermore, the membrane form of the Klotho protein acts as a fibroblast growth factor 23 co-factor and is involved in several functions including the regulation of calcium/phosphate and vitamin D metabolism $(2,3,6,7)$. However, the findings of recent studies demonstrated that the performance of the soluble form of Klotho varies from the membrane form and includes regulating multiple ion channels $(2,8,9)$, down-regulating Wnt, tumour necrosis factor alpha (TNF $\alpha)$, transforming growth factor beta (TGF $\beta)$, Interferon gamma (IFN $\gamma$ ) signaling, decreasing inflammation $(3,5,10,11)$, regulating IGF-1/ insulin signaling, attenuating reactive oxygen species and thus the suppression of oxidative stress, and increasing the antioxidant defense, anti-aging action, and NO production in endothelium $(2,9-12)$. A decrease in the 
circulating Klotho levels occurs in chronic kidney disease, cardiovascular disease (12-14), hypertensive and diabetic patients, and even normal aging $(1,4,5)$. Therefore, it can be associated with functional and cognitive impairment, sarcopenia (7), endothelial dysfunction, vascular calcification, atherosclerosis, and osteoporosis $(1,14)$. These findings suggest that reducing the levels of soluble Klotho can be a primary biomarker for diagnosing various cardiovascular disorders and pathological conditions (15). However, the mechanisms by which Klotho improves the function of various tissues is somehow unclear while recent studies indicated that this can be related to the lower levels of stress responses (5).

Considering the importance of Klotho, an effort to identify the interventional factors for its alteration and the discovery of its physiological and biochemical functions are of great importance. Additionally, regarding the role of exercise training and its similar effects, along with Klotho on antiaging, antioxidant, and antiapoptotic pathways (16-18), it seems that exercise training is one of the most effective applied approaches which has a positive and a protective role through increasing Klotho, followed by an increase in the antioxidant capacity and a decrease in oxidative stress. However, studies about changes in this protein following exercise training and its effective mechanisms are limited and therefore, further studies are needed to understand the mechanisms involved in increasing the Klotho by exercise training.

In addition, determining the types, duration, and intensity of effective exercise training is required to increase its positive outcomes. The intensity of exercise training is considered an important factor in the effectiveness of the exercise training program and many studies represented that cardiovascular, muscular, and metabolic adaptations in healthy and patient populations depend on the intensity of exercise training. These studies $(19,20)$ reported more beneficial effects of high-intensity interval training (HIIT) compared to moderate-intensity continuous training (MICT). Further, it was found that even short-term training sessions (e.g., 3-5 sessions) can improve molecular and physiological adaptation and increase Cardioprotection (21-24). However, despite limited evidence that indicates the positive impact of moderate-intensity aerobic training on the Klotho levels $(11,18,25)$, it is unclear whether or not high-intensity interval aerobic training can have such an advantage. Or is there any difference between these types of training programs? Investigating the effects of these exercise training, as well as recognizing the role of skeletal muscles as the regulators of Klotho is important and can lead to the development of physical activities and prevention and rehabilitation programs in order to counteract the effects of aging and disease. Therefore, due to the importance of selecting the correct and appropriate training programs, the current study sought to evaluate and compare the effects of short-term HIIT and MICT on the plasma levels of Klotho, antioxidant capacity, and lipid peroxidation.

\section{Materials and Methods}

Animals and experimental design

A total of 24 male Wistar rats (8-10 weeks old) with a weight range of 250-300 $\mathrm{g}$ were purchased from the Pasteur Institute of Iran (Tehran, Iran). After adaptation to the new standard environment for at least 10 days (temperature of $22 \pm 2^{\circ} \mathrm{C}$, relative humidity of $55 \%, 12: 12$ light-dark cycle, and free access to water and food), the rats were randomly categorized into control, HIIT and MICT groups. At the beginning of the study, the training groups were homogenized based on $\mathrm{VO}_{2}$ max.

\section{Exercise protocols}

After a familiarization period (3-4 days) on the treadmill at a gradual increase in running times and speeds, rats performed a $\mathrm{VO}_{2}$ max test according to the protocols of previous studies $(26,27)$. Then, after 72 hours of rest, the animals conducted 5 consecutive days of exercise training. The HIIT sessions included 5 minutes warm-up $(50 \%$ $\left.\mathrm{Vo}_{2} \max \right), 6 \times 2$ minutes high intermittence with $85-90 \%$ $\mathrm{Vo}_{2} \max$ and $5 \times 2$ minutes slow intermittence (active recovery) with $55 \%-60 \% \mathrm{Vo}_{2} \max$ and 5 minutes cool down $\left(50 \% \mathrm{Vo}_{2} \max \right)$. Furthermore, the training program was performed based on the pilot study and the changes in the intensity of the training protocol implemented in previous research (21). Additionally, the intensity of exercise training was modified based on previous studies $(26,27)$.

The MICT program included 5-minute warm-up (50\% $\mathrm{Vo}_{2} \max$ ), then running on the treadmill at $70 \% \mathrm{VO}_{2} \max$ (28) that total distance running was the same as the HIIT group, and finally, 5 minutes cool down (50\% $\left.\mathrm{Vo}_{2} \max \right)$.

The control group participated in no exercise program while they were placed on the immobile treadmill for 1520 minutes per session to create the same conditions for all the subjects

\section{Klotho, TAC, and MDA plasma level determination}

After 24 hours from the training period, blood samples were collected from the heart of the rats after anesthesia and poured into EDTA tubes in order to prevent clots. Then, the samples were centrifuged at $3000 \mathrm{rpm}$, for 20 minutes and plasma was separated. Next, the plasma level of Klotho was determined by Rat Klotho enzymelinked immune sorbent assay (ELISA) kit based on the Biotin double antibody sandwich technology (Cat. NO: E1206Ra, BT: bioassay technology laboratory, Shanghai crystal day biotech Co, LTD, Shanghai, China). In addition, in order to measure plasma levels of total antioxidant capacity (TAC) and malondialdehyde (MDA), ZellBio $\mathrm{GmbH}$ assay kits were used including MDA/TBARS assay kit based on the colorimetric method 
[Cat No. ZB-MDA96A, V405], and TAC kit based on the oxidation-reduction colorimetric assay [Cat No. ZBTAC96 ZellBio GmbH, Germany].

\section{Statistical analysis}

After determining data normality, one-way ANOVA and post-hoc Tukey tests were employed for analyzing the data and investigating the differences between the groups. Further, the Pearson correlation was used to determine the correlation between Klotho with TAC and MDA. Eventually, the obtained data were analyzed by the SPSS software, version 16. All data were presented as means \pm SD and $P<0.05$ was considered statistically significant in all tests.

\section{Results}

There was no significant difference between the groups concerning the weight at the beginning of the training (Table 1). As displayed in Figure 1, the results of this study indicate a significant increase in plasma levels of Klotho after both HIIT and MICT $(2.34 \pm 0.34$ and $1.94 \pm 0.28$, respectively, $P<0.001)$. However, the increase in Klotho is significantly higher in HIIT group compared to MICT group $(P=0.024)$. Furthermore, based on the data illustrated in Figure 2, the TAC significantly increases after both of the training protocols compared to the control group $(0.31 \pm 0.031$ in HIIT and $0.27 \pm 0.029$ in MICT groups vs. $0.22 \pm 0.033$ in control group; $P<0.001$ ) whereas it is significantly higher in the HIIT group

Table 1. The Body Weight of Rats in Experimental Groups

\begin{tabular}{ccccc}
\hline & $\begin{array}{c}\text { Control } \\
\text { Mean } \pm \text { SD }\end{array}$ & $\begin{array}{c}\text { HIIT } \\
\text { Mean } \pm \text { SD }\end{array}$ & $\begin{array}{c}\text { MICT } \\
\text { Mean } \pm \text { SD }\end{array}$ & $\boldsymbol{P}$ \\
\hline Body weight $(\mathrm{g})$ & $282.57 \pm 13.04$ & $275.12 \pm 14.31$ & $278.53 \pm 13.37$ & 0.557 \\
\hline
\end{tabular}

Abbreviation: HIIT: High intensive internal training; MICT: Moderate intensity continuous training

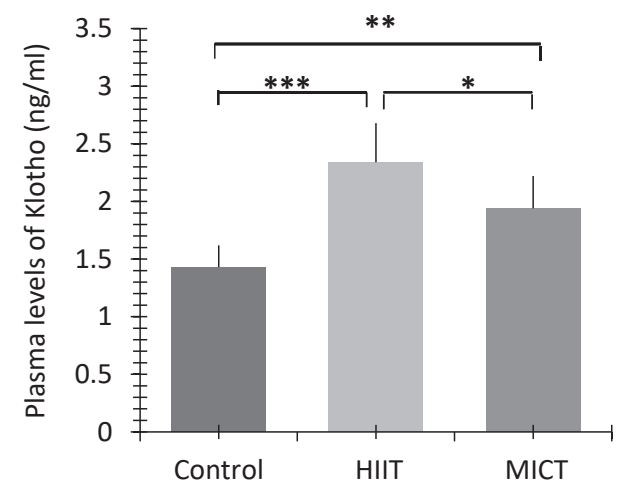

Figure 1. Plasma Levels of Klotho in experimental groups. Plasma levels of Klotho in both training groups are significantly higher compared to the control group. In addition, the increase in Klotho is significantly higher in the HIIT group compared to MICT group. Data are expressed as mean \pm SD. The level of significance between the two compared groups are ${ }^{*} P=0.024$, $* * P=0.004$, and ${ }^{* * *} P=0.0001$ compared to MICT $(P=0.033)$. Contrarily, as depicted in Figure 3, there is no significant difference between the groups respecting MDA $(P=0.109)$. Additionally, the results reveal a significant positive correlation between Klotho and TAC whereas a negative correlation between Klotho with MDA among the groups. The results of the correlation are represented in Table 2 .

\section{Discussion}

The results of the present study indicated that even short-term HIIT and MICT increase plasma levels of Klotho although a significant difference between these two training groups represents a greater effect of HIIT compared to MICT. The result of the current study is in line with the results of Avin et al (25) and Matsubara et al (11) who found a positive effect of aerobic training on the blood levels of Klotho. In addition, the results of research indicating the higher levels of Klotho in healthy trained groups compared to the untrained groups $(12,18,29)$ can confirm the positive effect of aerobic training on the blood levels of Klotho. However, despite the evidence that demonstrates the positive effects of aerobic training on the levels of Klotho in young and old healthy $(11,18,25)$, as well as patients with heart disease (30), no research was

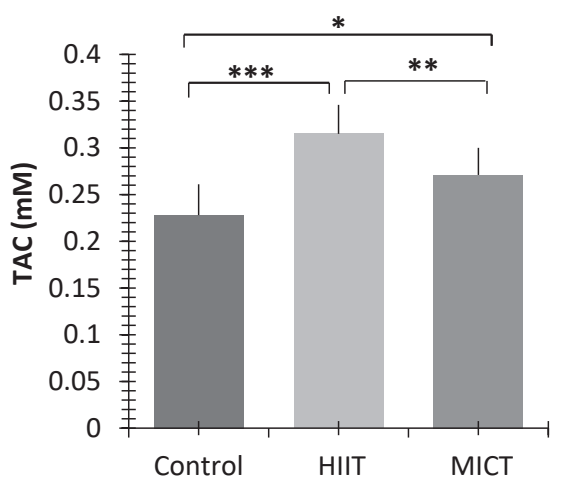

Figure 2. Plasma Levels of TAC in experimental groups. Plasma levels of TAC in both training groups are significantly higher compared to the control group. Further, TAC in HIIT group is significantly higher than that of MICT group. Data are expressed as mean $\pm \mathrm{SD}$. The level of significance between the two compared groups are ${ }^{*} P=0.033,{ }^{* *} P=0.028$ and ${ }^{* * *} P=0.0001$

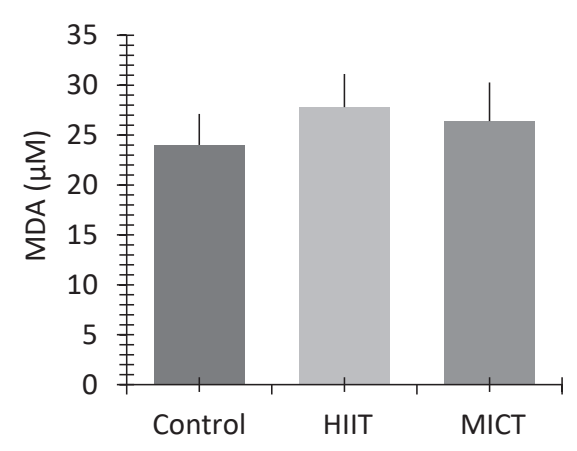

Figure 3. Plasma Levels of MDA in experimental groups. There is no significant difference between the groups $(P=0.109)$. Data are expressed as mean \pm SD 
Table 2. Correlation between circulating levels of Klotho, TAC, and MDA in the groups

\begin{tabular}{|c|c|c|c|c|c|c|c|c|c|}
\hline & \multicolumn{3}{|c|}{ Control } & \multicolumn{3}{|c|}{ HIIT } & \multicolumn{3}{|c|}{ MICT } \\
\hline & Klotho & TAC & MDA & Klotho & TAC & MDA & Klotho & TAC & MDA \\
\hline Klotho & - & $\begin{array}{c}r=0.79 \\
P=0.018\end{array}$ & $\begin{array}{l}r=-0.78 \\
P=0.022\end{array}$ & - & $\begin{aligned} \mathrm{r} & =0.83 \\
P & =0.010\end{aligned}$ & $\begin{array}{l}r=-0.86 \\
P=0.005\end{array}$ & - & $\begin{array}{c}r=0.79 \\
P=0.018\end{array}$ & $\begin{array}{l}\mathrm{r}=-0.81 \\
P=0.014\end{array}$ \\
\hline TAC & $\begin{array}{c}r=0.79 \\
P=0.018\end{array}$ & - & $\begin{array}{l}r=-0.73 \\
P=0.038\end{array}$ & $\begin{array}{c}r=0.83 \\
P=0.010\end{array}$ & - & $\begin{array}{l}r=-0.80 \\
P=0.015\end{array}$ & $\begin{array}{c}r=0.79 \\
P=0.018\end{array}$ & - & $\begin{array}{l}r=-0.87 \\
P=0.005\end{array}$ \\
\hline MDA & $\begin{array}{l}r=-0.78 \\
P=0.022\end{array}$ & $\begin{array}{l}r=-0.73 \\
P=0.038\end{array}$ & - & $\begin{array}{l}r=-0.86 \\
P=0.005\end{array}$ & $\begin{array}{l}r=-0.80 \\
P=0.015\end{array}$ & - & $\begin{array}{l}r=-0.81 \\
P=0.014\end{array}$ & $\begin{array}{l}r=-0.87 \\
P=0.005\end{array}$ & - \\
\hline
\end{tabular}

conducted in the field of different forms and intensities of training regarding investigating its greater effectiveness. There is only one study, to the best of our knowledge, which compared Klotho levels between aerobic and anaerobic athletes and the results revealed that, unlike aerobic exercise, an anaerobic exercise is not considered a strong stimulus to increase soluble Klotho levels (31).

Due to the limited number of studies respecting the effect of exercise training on the expression of Klotho protein, the mechanisms responsible for the increase in soluble Klotho following exercise training have not yet been elucidated. As a result, it is not yet clear whether muscle contraction increases the expression and local production of this protein or some myokines increase the expression of this protein in other tissues such as the kidneys and brain (25). Changes in circulating concentrations of Klotho may be due to the increase in the secreted form of Klotho or an increase in the degradation of the extracellular domain of its membrane form. Therefore, aerobic training is likely to increase the soluble Klotho concentration through the intervention in these pathways while its mechanisms are still unknown (11).

Further, factors such as an increase in the transcription factor of peroxisome proliferator-activated receptor $\gamma$ (PPAR- $\gamma$ ), a decrease in angiotensin II type I receptor signaling, and the reduction of oxidative stress following exercise training affect the increase in the expression of Klotho mRNA and protein (11). Accordingly, the increase in circulating Klotho is probably the result of the abovementioned factors. However, further research is needed to understand the effective mechanisms.

Furthermore, based on the findings of the present study, it is likely that more stimulation of peroxisome proliferatoractivated receptor gamma coactivator 1-alpha (PGC1 $\alpha$ ) and PPAR- $\gamma$ expression and maybe more hypoxia following HIIT are regarded as a greater stimulus to increase the Klotho compared to MICT. However, the expression of PGC1 $\alpha$ and PPAR- $\gamma$ have not been investigated and need to be studied. Additionally, due to different cardiovascular, muscular and metabolic responses, and adaptations of these two protocols, probably different patterns in blood flow and shear stress, changes in vasodilation and endothelial biomarkers, and an increase in antioxidant capacity following exercise training can be considered the mechanisms of more prominent changes following HIIT compared to the MICT. However, different training protocols and possible mechanisms should be investigated in order to clarify this effect and its related mechanisms.

Various studies indicated that soluble Klotho can inhibit the phosphorylation of IGF1/insulin receptors and downstream signaling under physiological conditions. Thus, soluble Klotho inhibits Forkhead box O (i.e., FOXO, transcription factors) phosphorylation while it increases its activity which leads to an increase in $\mathrm{Mn}$ SOD expression and antioxidant enzymes, and ultimately facilitates the removal of reactive oxygen species, increases resistance to oxidative stress, and protects the tissues $(2,32)$. In addition, Klotho increases FOXO3a (i.e., a negative regulator of mitochondrial ROS synthesis) and can inhibit the oxidative stress while its deficiency increases the production of endogenous ROS and oxidative stress $(5,32)$. Therefore, although the mechanisms through which Klotho improves the function of different tissues is not completely clear, recent studies suggest that it can be related to lower levels of the stress response and resistance to oxidative stress $(2,5)$. Several studies (32-34) reported that Clotho reduces oxidative stress, the expression of Bax protein, apoptosis, mitochondrial DNA fragmentation, superoxide synthesis, lipid peroxidation, NADPH oxidase-2 activity (Nox2), and 8-OHdG (DNA oxidative marker). Further, the evidence indicates that klotho plays a protective role and increases resistance against oxidative stress by activating the AMP signaling, producing NO, as well as increasing SOD expression and antioxidant capacity (35). In the present study, the increase in TAC and its association with the enhancement of Klotho and correlation between Klotho and TAC and MDA in study groups can confirm these findings and highlight the role of Klotho as a regulator of oxidative stress.

Furthermore, the intensity and duration of exercise are important variables which can interfere with oxidative stress and antioxidant status. Research revealed that exercise, especially long and intense exercise can increase ROS, oxidative stress, and lipid peroxides enhancing the activity of catecholamines, prostanoids, NADP $(\mathrm{H})$ oxidase, the activity of macrophages (36), oxidative phosphorylation, the rate of oxygen exchange in the electron transport chain $(37,38)$, while reducing local 
blood flow to the muscles at the beginning of the activity, namely, ischemia-reperfusion process in the muscles (38). However, there are some studies which emphasize the role of regular aerobic exercises, even HIIT, controlling the oxidative stress indices and preventing lipid peroxidation $(39,40)$. Based on the findings of the current study, despite an increase in MDA in the training groups, no statistically significant difference was observed between the groups after 24 hours. These results demonstrated that exercise training could make adaptations to attenuate exerciseinduced oxidative stress and lipid peroxidation. Moreover, the increase in Klotho and antioxidant defense following exercise training can remove active oxygen species produced by the exercise training and confer resistance against oxidative stress. Therefore, it can be claimed that even short-term aerobic exercises (e.g., HIIT and MICT) created antioxidant adaptations which increased TAC in order to cope with oxidative stress.

The results of this study stressed the role of aerobic training in enhancing antioxidant capacity and controlling oxidative stress indices. Additionally, mechanisms responsible for the increase in the amount and activity of the antioxidant system following exercise training can include the increase in adenosine as a result of ATP consumption (41), as well as the activation of MAPK (adenosine monophosphateactivated protein kinase), NF- $\kappa \mathrm{B}$ (transcription factors) pathway, and FoxO transcription factor (42). In addition, exercise-induced ROS production acts as the signals which activate MAPKs (p38 and ERK1/2) and thus, activate NF$\kappa \mathrm{B}$ and upregulate the expression of antioxidant enzymes (43). Further, based on the results of the present study, the increase in Klotho levels after exercise training can be an effective mechanism since Klotho has antioxidant properties and can act as a protective protein by activating the FOXO and increasing antioxidant enzymes, especially SOD $(31,32)$. As a result, the increase in TAC and the relationship between Klotho and TAC following exercise training can confirm the effect of Klotho on increasing the antioxidant capacity and positive results of exercise training.

\section{Conclusion}

In general, the findings of the current study suggest that even short-term aerobic exercise training (both HIIT and MICT) increases plasma levels of Klotho and antioxidant capacity and regulates oxidative stress. Therefore, it can have a positive effect on the body since an increase in this protein has clinical importance in the health of organs and the pathophysiology of various diseases. Although the result of this study helps to clarify the further effect of HIIT compared to MICT, different training protocols and possible mechanisms should be investigated to identify such an effect in order to select the appropriate exercise training program.
Conflict of interests

None.

Ethical considerations

The present study was performed according to the guidelines for the care and use of laboratory animals published by the US national institutes of health (NIH Publication No. 85-23, revised in 1996). All experimental procedures were approved by the Animal Ethics Committee of Iran University of Medical Sciences (IR.IUMS.REC 1395.28040) and the study was implemented in the animal lab of Rajaie Cardiovascular Medical and Research Center and Iran University of Medical Sciences (Tehran, Iran).

\section{Acknowledgments}

This study was part of the thesis submitted by the first author and conducted in Iran University of Medical Sciences, as well as Rajaie Cardiovascular Medical and Research Center, Tehran, Iran (code 28040). We greatly appreciate all those who helped us somehow in this research.

\section{References}

1. Martin-Nunez E, Donate-Correa J, Muros-de-Fuentes M, Mora-Fernandez C, Navarro-Gonzalez JF. Implications of Klotho in vascular health and disease. World J Cardiol. 2014;6(12):1262-9. doi: 10.4330/wjc.v6.i12.1262.

2. Kuro-o M. Klotho and aging. Biochim Biophys Acta. 2009;1790(10):1049-58. doi: 10.1016/j.bbagen.2009.02.005.

3. Kim JH, Hwang KH, Park KS, Kong ID, Cha SK. Biological Role of Anti-aging Protein Klotho. J Lifestyle Med. 2015;5(1):1-6. doi: 10.15280/jlm.2015.5.1.1.

4. Maltese G, Karalliedde J. The putative role of the antiageing protein klotho in cardiovascular and renal disease. Int J Hypertens. 2012;2012:757469. doi: 10.1155/2012/757469.

5. Xu Y, Sun Z. Molecular basis of Klotho: from gene to function in aging. Endocr Rev. 2015;36(2):174-93. doi: 10.1210/ er.2013-1079.

6. Kuro-o M. Klotho. Pflugers Arch. 2010;459(2):333-43. doi: 10.1007/s00424-009-0722-7.

7. Moe SM. Klotho: a master regulator of cardiovascular disease? Circulation. 2012;125(18):2181-3. doi: 10.1161/ circulationaha.112.104828.

8. Huang CL. Regulation of ion channels by secreted Klotho: mechanisms and implications. Kidney Int. 2010;77(10):85560. doi: 10.1038/ki.2010.73.

9. Pavlatou MG, Remaley AT, Gold PW. Klotho: a humeral mediator in CSF and plasma that influences longevity and susceptibility to multiple complex disorders, including depression. Transl Psychiatry. 2016;6(8):e876. doi: 10.1038/ tp.2016.135.

10. Wang Y, Sun Z. Current understanding of klotho. Ageing Res Rev. 2009;8(1):43-51. doi: 10.1016/j.arr.2008.10.002.

11. Matsubara T, Miyaki A, Akazawa N, Choi Y, Ra SG, Tanahashi $K$, et al. Aerobic exercise training increases plasma Klotho levels and reduces arterial stiffness in postmenopausal women. Am J Physiol Heart Circ Physiol. 2014;306(3):H34855. doi: 10.1152/ajpheart.00429.2013.

12. Lim K, Lu TS, Molostvov G, Lee C, Lam FT, Zehnder D, et al. Vascular Klotho deficiency potentiates the development of human artery calcification and mediates resistance to fibroblast growth factor 23. Circulation. 2012;125(18):224355. doi: 10.1161/circulationaha.111.053405.

13. Tanaka S, Fujita S, Kizawa S, Morita H, Ishizaka N. Association between FGF23, alpha-Klotho, and Cardiac Abnormalities among Patients with Various Chronic Kidney Disease Stages. PLoS One. 2016;11(7):e0156860. doi: 10.1371/journal. pone. 0156860 .

14. Lindberg K, Olauson H, Amin R, Ponnusamy A, Goetz $\mathrm{R}$, Taylor RF, et al. Arterial klotho expression and FGF23 
effects on vascular calcification and function. PLoS One. 2013;8(4):e60658. doi: 10.1371/journal.pone.0060658.

15. Zhou X, Li S, Wang Z, Yu L, Jiang H. Klotho protein: A potential therapeutic agent during myocardial ischemia and reperfusion. Int J Cardiol. 2015;191:227-8. doi: 10.1016/j. ijcard.2015.05.029.

16. Kwak HB. Effects of aging and exercise training on apoptosis in the heart. J Exerc Rehabil. 2013;9(2):212-9. doi: 10.12965/ jer.130002.

17. Lira-Ferrari GS, Bucalen-Ferrari CK. Exercise modulation of total antioxidant capacity (TAC): towards a molecular signature of healthy aging. Front Life Sci. 2011;5(3-4):81-90.

18. Ben-Sira D, Goldhammer E, Saghiv MS, Saghiv M. Effects of Aerobic Exercise Training on S-Klotho in Young and Elderly. Jacobs J Physiol. 2015;1(1):001.

19. Guiraud T, Nigam A, Gremeaux V, Meyer P, Juneau M, Bosquet L. High-intensity interval training in cardiac rehabilitation. Sports Med. 2012;42(7):587-605. doi: 10.2165/11631910000000000-00000.

20. Libonati JR, Kendrick ZV, Houser SR. Sprint training improves postischemic, left ventricular diastolic performance. J Appl Physiol (1985). 2005;99(6):2121-7. doi: 10.1152/ japplphysiol.01212.2004.

21. Rahimi M, Shekarforoush S, Asgari AR, Khoshbaten A, Rajabi $\mathrm{H}$, Bazgir B, et al. The effect of high intensity interval training on cardioprotection against ischemia-reperfusion injury in wistar rats. Excli j. 2015;14:237-46. doi: 10.17179/excli2014-587.

22. French JP, Hamilton KL, Quindry JC, Lee $\mathrm{Y}$, Upchurch PA, Powers SK. Exercise-induced protection against myocardial apoptosis and necrosis: MnSOD, calcium-handling proteins, and calpain. FASEB J. 2008;22(8):2862-71. doi: 10.1096/ fj. 07-102541.

23. Kavazis AN, McClung JM, Hood DA, Powers SK. Exercise induces a cardiac mitochondrial phenotype that resists apoptotic stimuli. Am J Physiol Heart Circ Physiol. 2008;294(2):H928-35. doi: 10.1152/ajpheart.01231.2007.

24. Lee Y, Min K, Talbert EE, Kavazis AN, Smuder AJ, Willis WT, et al. Exercise protects cardiac mitochondria against ischemiareperfusion injury. Med Sci Sports Exerc. 2012;44(3):397-405. doi: 10.1249/MSS.0b013e318231c037.

25. Avin KG, Coen PM, Huang W, Stolz DB, Sowa GA, Dube $\mathrm{JJ}$, et al. Skeletal muscle as a regulator of the longevity protein, Klotho. Front Physiol. 2014;5:189. doi: 10.3389/ fphys.2014.00189.

26. Hoydal MA, Wisloff $U$, Kemi OJ, Ellingsen O. Running speed and maximal oxygen uptake in rats and mice: practical implications for exercise training. EurJ Cardiovasc Prev Rehabil. 2007;14(6):753-60. doi: 10.1097/HJR.0b013e3281eacef1.

27. Kemi OJ, Haram PM, Loennechen JP, Osnes JB, Skomedal T, Wisloff $\mathrm{U}$, et al. Moderate vs. high exercise intensity: differential effects on aerobic fitness, cardiomyocyte contractility, and endothelial function. Cardiovasc Res. 2005;67(1):161-72. doi: 10.1016/j.cardiores.2005.03.010.

28. Haram PM, Kemi OJ, Lee SJ, Bendheim MO, Al-Share QY, Waldum $\mathrm{HL}$, et al. Aerobic interval training vs. continuous moderate exercise in the metabolic syndrome of rats artificially selected for low aerobic capacity. Cardiovasc Res. 2009;81(4):723-32. doi: 10.1093/cvr/cvn332.

29. Mostafidi E, Moeen A, Nasri H, Ghorbani Hagjo A, Ardalan M. Serum Klotho Levels in Trained Athletes. Nephrourol Mon. 2016;8(1):e30245. doi: 10.5812/numonthly.30245.

30. Saghiv MS, Sherve C, Ben-Sira D, Sagiv M, Goldhamme E. Aerobic Training Effect on Blood S-Klotho Levels in Coronary Artery Disease Patients. J Clin Exp Cardiolog. 2016;7(8):1-4. doi: 10.4172/2155-9880.1000464.

31. Saghiv MS, Ben-Sira D, Sagiv M. Comparison between aerobic and anaerobic training influence on s-klotho blood levels following 60 min aerobic bout. J Physiology. 2015;1(1):003.

32. Yamamoto M, Clark JD, Pastor JV, Gurnani P, Nandi A, Kurosu $\mathrm{H}$, et al. Regulation of oxidative stress by the anti-aging hormone klotho. J Biol Chem. 2005;280(45):38029-34. doi: 10.1074/jbc.M509039200.

33. Wang $Y$, Kuro-o M, Sun Z. Klotho gene delivery suppresses Nox2 expression and attenuates oxidative stress in rat aortic smooth muscle cells via the cAMP-PKA pathway. Aging Cell. 2012;11(3):410-7. doi: 10.1111/j.1474-9726.2012.00796.x.

34. Haruna $Y$, Kashihara N, Satoh $M$, Tomita N, Namikoshi T, Sasaki $\mathrm{T}$, et al. Amelioration of progressive renal injury by genetic manipulation of Klotho gene. Proc Natl Acad Sci U S A. 2007;104(7):2331-6. doi: 10.1073/pnas.0611079104.

35. Kuro-o M. Klotho as a regulator of oxidative stress and senescence. Biol Chem. 2008;389(3):233-41. doi: 10.1515/ bc.2008.028.

36. Clarkson PM, Thompson HS. Antioxidants: what role do they play in physical activity and health? Am J Clin Nutr. 2000;72(2 Suppl):637s-46s. doi: 10.1093/ajcn/72.2.637S.

37. Blokhina O, Virolainen E, Fagerstedt KV. Antioxidants, oxidative damage and oxygen deprivation stress: a review. Ann Bot. 2003;91 Spec No:179-94.

38. Cooper CE, Vollaard NB, Choueiri T, Wilson MT. Exercise, free radicals and oxidative stress. Biochem Soc Trans. 2002;30(2):280-5. doi: 10.1042/bst0300280.

39. Sandeep Kumar S, Hassan MA. Effect of high intensity interval training on malondialdehyde and aerobic capacity of male physical education students. Int J Curr Adv Res. 2013;2(1):579.

40. Cunningham P, Geary M, Harper R, Pendleton A, Stover S. High intensity sprint training reduces lipid peroxidation in fasttwitch skeletal muscle. J Exerc Physiol Online. 2005;8(6):1825.

41. Pinho RA, Andrades ME, Oliveira MR, Pirola AC, Zago MS, Silveira PC, et al. Imbalance in SOD/CAT activities in rat skeletal muscles submitted to treadmill training exercise. Cell Biol Int. 2006;30(10):848-53. doi: 10.1016/j.cellbi.2006.03.011.

42. Steinbacher P, Eckl P. Impact of oxidative stress on exercising skeletal muscle. Biomolecules. 2015;5(2):356-77. doi: 10.3390/biom5020356.

43. Fisher-Wellman K, Bell HK, Bloomer RJ. Oxidative stress and antioxidant defense mechanisms linked to exercise during cardiopulmonary and metabolic disorders. Oxid Med Cell Longev. 2009;2(1):43-51. 